\title{
Study on Embedded CNC System for NURBS Curves Method of Interpolation Arithmetic
}

\author{
Wanjun Zhang 1, 2, 3, a, Shanping Gao 1, b, Xiyan Cheng 1, c, Feng Zhang 2, d \\ ${ }^{1}$ Quanzhou Institute of Information Engineering, 36200, China \\ ${ }^{2}$ School of Mechanical Engineering, Xian Jiao tong University, 741049, China \\ ${ }^{3}$ Lanzhou Industry and Equipment Co. Ltd, Lanzhou 730050, China \\ agszwj_40@163.com, b358391056@qq.com, c326593363@qq.com, d116543048@qq.com
}

Keywords: NURBS Curves; Interpolation Arithmetic; Embedded CNC System; PID Control.

\begin{abstract}
In order to reduce the calculation amount of NURBS curves, combined with NURBS curves mathematical properties. Firstly this paper introduce on the algorithm of the NURBS curves improvement, it is the in the interpolation cycle under the condition of certain interpolation increment only and interpolation speed, by changing the interpolation increments can achieve correction curve of interpolation. Secondly, the control platform based on ARM+DSP could overcome the defects of CNC, through the design of hardware and software. Also, it realizes real-time dispatch and improves the reliability and stability. Lastly, on the basis of improvement PID numberical control arithmetic, this paper adopts Ziegler-Nichols theory to set PID parameters and uses it in embedded CNC system servo control. Also this paper users MATLAB/SIMULINk to do simulation and analyses about select. The simulation result showed that the correction by using PID controller, to servo system for real-time control, system stability improvement at the same time, response speed, feed system and raises the comprehensive performance.
\end{abstract}

\section{Introduction}

For complex curves and surface treatment, the calculation of NURBS curve is large, which will affect the response time of motion seriously. In order to reduce the computational complexity, the NURBS curve is mainly studied. When the feature points or points than the distance between the three and the last, the NURBS curve of the difference is very small and simple) [1], so the NURBS curve is on the curve structure method and engineering and technical personnel to solve the relevant problems of choice.

Research on NURBS curve interpolation algorithm has a large number of documents, many of which are in order to produce fast algorithm of curve on the computer and the design of the NURBS curve, most of the solutions of triangular equations, parallel algorithms such as recursive coupling, cyclic reduction method, matrix decomposition method, but for multi core computer all applicable [2]. Zhao Guoyong et al. A fast and real time interpolation algorithm for NURS curve interpolation in high speed and high precision NC machining [3]. [4-8] curve parameters adaptive interpolation algorithm, improved algorithm, parallel machine tool interpolation algorithm is proposed, which improves the approximation error of NURBS. The interpolation algorithm [9-10] given a curve improvement and correction curve, Taylor formula of first order in the expansion, the increment interpolation and interpolation speed, the derivative can achieve the purpose of curve interpolation. Although the above algorithm can obtain a constant speed, without considering the error control. For the interpolation algorithm of NURBS curve interpolation, Taylor's expansion first order and two order solution is more complicated, and the processing error is larger.

Finally, through the research on the NURBS curve interpolation algorithm, NURBS curve interpolation calculation and general computational complexity, proposed an improved interpolation algorithm, can reduce the time and improve the efficiency of interpolation interpolation. The improved method is given by using MATLAB simulation servo system PID algorithm, the results show that the 
interpolation algorithm can reduce interpolation computation and interpolation operation debugging time and reduce the overshoot, it has strong adaptability and versatility, provides a new method for complex dynamic control of uncertain systems.

\section{Improved of NURBS curve interpolation algorithm}

\subsection{Mathematical foundation of NURBS curve}

In this paper, NURBS curve is used to represent a parametric of an improved algorithm adaptive of NURBS curve, and it is introduced first. Supposed $p(u)$ can be represented a Improved algorithm adaptive of NURBS curve. While NURBS [3] are parametrically mathematical definition by the following Eq.(1):

$$
p(u)=\frac{\sum_{i=0}^{n} \omega_{i} d_{i, k}(u)}{\sum_{i=0}^{n} \omega_{i} N_{i, k}(u)}=\sum_{i=0}^{n} p_{i} N_{i, k}(u)
$$

Where $u$ is cubic time an improved algorithm adaptive of NURBS curve each parameter, $\mathrm{k}$ the order of an improved algorithm adaptive of NURBS curve. $p_{i}$ is the control points, $\omega_{i}$ is the weight vector, $N_{i, k}(u)$ is the blending function.

$$
N_{i, k}(u)=\left\{\begin{array}{c}
N_{i, k}=\left\{\begin{array}{l}
1, \boldsymbol{u}_{i} \leq \boldsymbol{u} \leq \boldsymbol{u}_{i+1} \\
0, \text { other }
\end{array}\right. \\
\boldsymbol{N}_{i, k}=\frac{\boldsymbol{u}-\boldsymbol{u}_{i}}{\boldsymbol{u}_{i+k+1}-\boldsymbol{u}_{i}} N_{i, k-1}(u) \\
\quad+\frac{\boldsymbol{u}_{i+k+1}-\boldsymbol{u}}{\boldsymbol{u}_{i+k+1}-\boldsymbol{u}_{i+1}} \\
\text { define }: \frac{0}{0}=0
\end{array}\right.
$$

By substituting $U$

$$
U=\{\underbrace{c, \cdots, c}_{p+1}, \boldsymbol{u}_{p+1}, \cdots, \boldsymbol{u}_{m-p-1}, \underbrace{d, \cdots, d}_{p+1}\}
$$

In this mathematical model of Newton-Rapson iterative interpolation algorithm, as shown in Fig.1.If we can suppose NURBS curve with existing maximum deceleration piont $p\left(u_{i}\right)$ and minimum acceleration piont $p\left(\boldsymbol{u}_{i+1}\right)$.

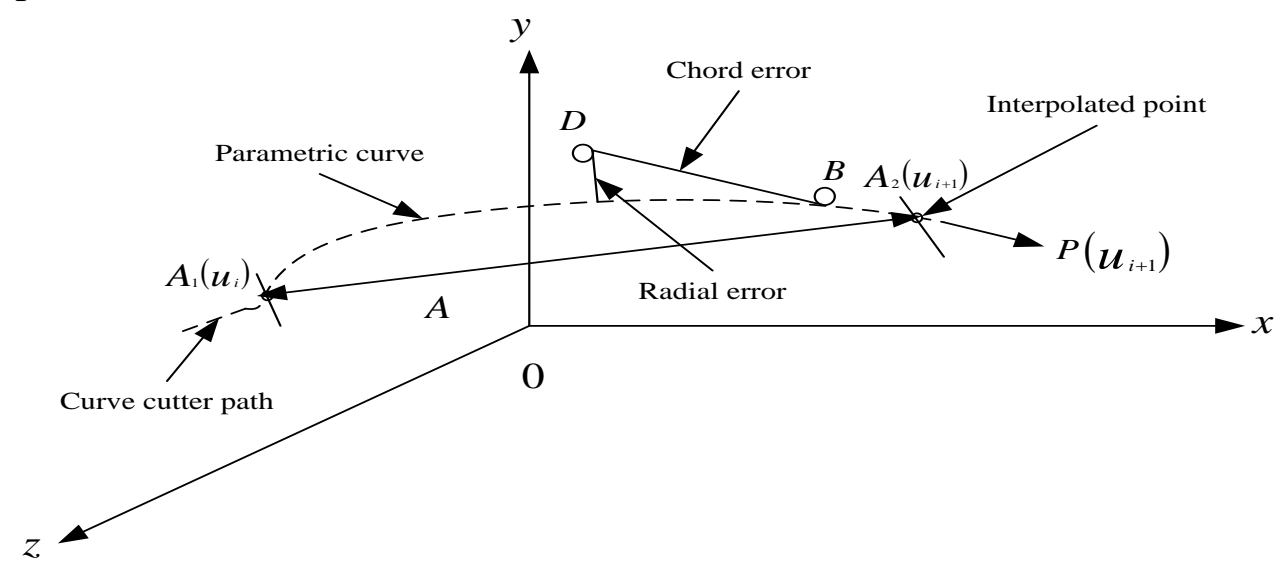

Fig. 1 This mathematical model of NURBS curve interpolation

Newton-Rapson iteration, Where

$$
u_{i+1}-\boldsymbol{u}_{i}=\frac{f\left(\boldsymbol{u}_{i}\right)}{f^{\prime}\left(\boldsymbol{u}_{i}\right)}, \quad i \geq 1
$$




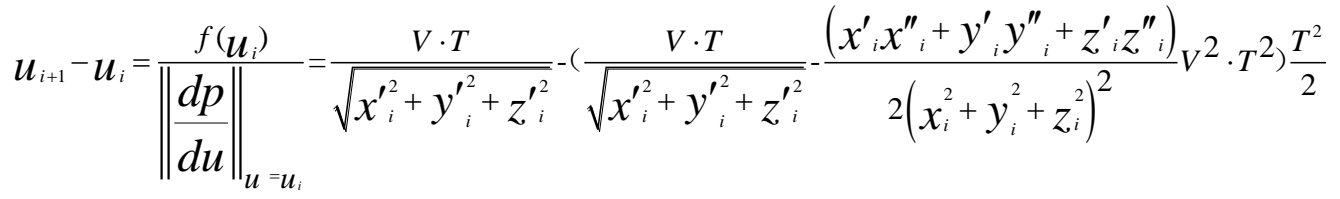

Newton-Rapson Cyclic iteration rule

$$
\frac{\left|u_{i+1}-u_{i}\right|}{u_{i}}<\varepsilon, \quad u_{i} \neq 0
$$

Where,

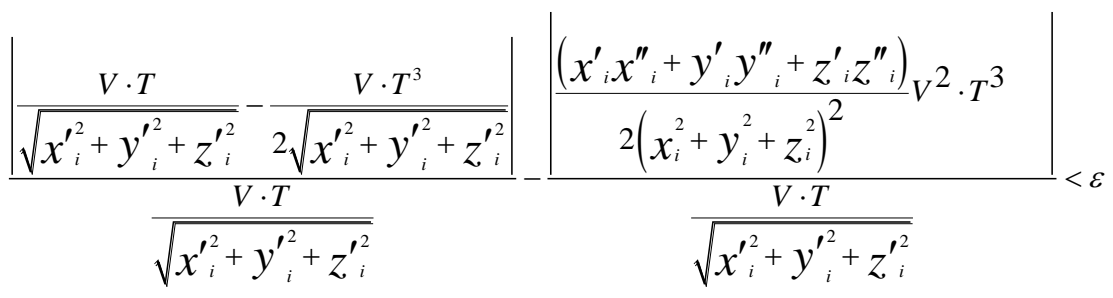

From the formula (1) (10), can be found out of the coordinates of the point, From the calculation method of the above view by two order Taylor for NURBS curve interpolation coordinates expansion and Newton-Rapson iterative method, interpolation calculation and interpolation process is complex, cumbersome, long processing time, interpolation interpolation inefficient and error accuracy is not high. Therefore, it can reduce the amount of interpolation, eliminate the cumulative error in the tangent direction of the NURBS curve, save the interpolation time and improve the efficiency of interpolation.

\section{2 improved interpolation algorithm}

By using the Taylor formula to simplify the NURBS curve interpolation, the formula (5) (6) by Taylor two order expansions to:

$$
u_{i+1}=u_{i}+\left.\frac{d u}{d t}\right|_{t=t_{i}} T+\left.\frac{1}{2} \frac{d^{2} u}{d t^{2}}\right|_{t=t_{i}} T^{2}+\frac{(\Delta T)^{3}}{3 !} f^{3}(\xi)
$$

Generally very small, so the two order Taylor formula in the truncation error, ignoring it does not affect the processing.

By the formula (3) (7) and (8) available, the NURBS curve of two order recursive formula

$$
u_{i+1}=u_{i}+\frac{\Delta L_{i}}{\sqrt{x^{2}+y^{2}+z^{2}}}-\frac{\Delta L_{i}^{2}\left(x^{\prime} x^{\prime \prime}+y^{\prime} y^{\prime \prime}+z^{\prime} z^{\prime \prime}\right)}{\left(x^{\prime 2}+y^{\prime 2}+z^{\prime 2}\right)}
$$

When ${ }^{\Delta} L_{i}$ compared to an hour, the available formula (13):

$$
u_{i+1} \approx u_{i}+\frac{\Delta L_{i}}{\left|\frac{d p(u)}{d t}\right|_{u}=u_{i}}=\frac{\Delta L_{i}}{\sqrt{x_{i}^{\prime 2}+y_{i}^{\prime 2}+z_{i}^{\prime 2}}} \text {. }
$$

The feed increment $x, y, z$ of each shaft is obtained by using the three coordinate axes control, and the coordinate position of the new interpolation point is obtained:

$$
\begin{aligned}
& X^{\prime}\left(u_{i}\right)=\frac{\Delta x}{\Delta u} \Rightarrow \Delta \boldsymbol{X}_{i}=X^{\prime}\left(u_{i}\right) \cdot \Delta u \\
& \left\{\begin{array}{l}
\Delta y_{i}=Y^{\prime}\left(u_{i}\right) \cdot \Delta u \\
\Delta z_{i}=Z^{\prime}\left(u_{i}\right) \cdot \Delta u
\end{array}\right. \\
& \Delta L=V \times T=\sqrt{\Delta x_{i}^{2}+\Delta y_{i}^{2}+\Delta z_{i}^{2}}=\Delta u \cdot \sqrt{\left[X\left(u_{i}\right)\right]^{2}+\left[Y\left(u_{i}\right)\right]^{2}+\left[Z\left(u_{i}\right)\right]^{2}} \\
& \Rightarrow \Delta u=\frac{V \times T}{\sqrt{\left[X\left(u_{i}\right)\right]^{2}+\left[Y\left(u_{i}\right)\right]^{2}+\left[Z\left(u_{i}\right)\right]^{2}}}
\end{aligned}
$$

So the next interpolation cycle is: 


$$
\left\{\begin{array}{l}
X_{i+1}=X\left(u_{i}+\Delta u\right) \\
Y_{i+1}=Y\left(u_{i}+\Delta u\right) \\
Z_{i+1}=Z\left(u_{i}+\Delta u\right)
\end{array}\right.
$$

Can be seen from the above formula (14), and related $\left(X_{i+1}, Y_{i+1}\right.$ and $\left.Z_{i+1}\right)$. Therefore, the key of CNC honing machine interpolation is realized by interpolation, increment in interpolation period, interpolation iterative fast and interpolations of each axis. According to the method of [4] and [8], the calculation is simplified, and the interpolation value of each axis $\mathrm{x}, \mathrm{y}, \mathrm{z}$ is calculated. The interpolation method, the calculation time is stable, in line with the actual production of interpolation needs, flow chart as shown in figure 1.

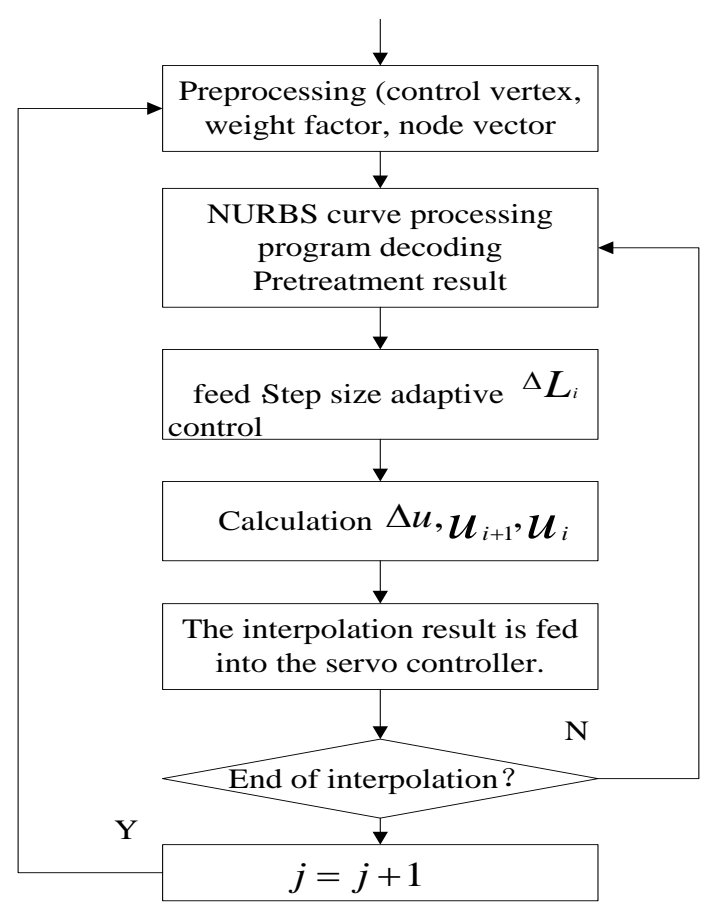

Fig. 2 Flow chart of NURBS curve interpolation algorithm

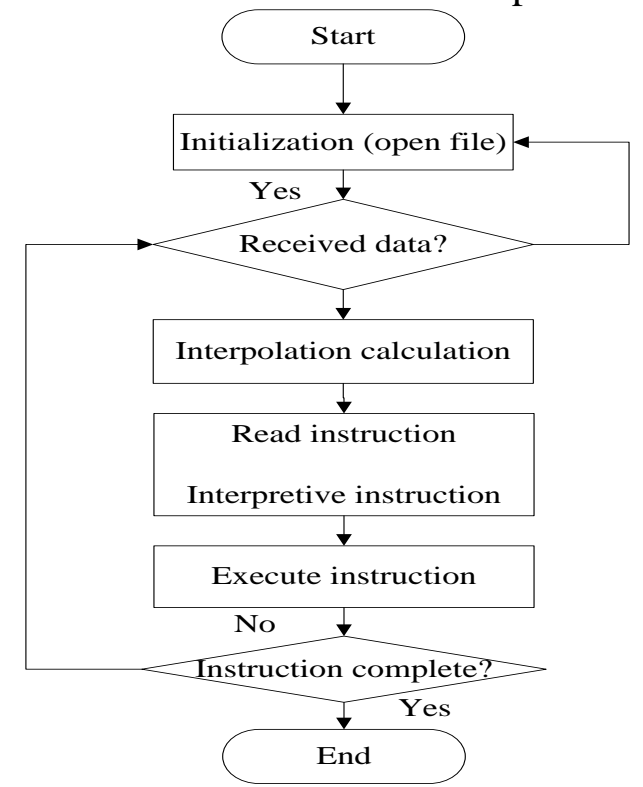

Fig. 3 Flow chart of system software design

\section{Design of embedded digital control system}

ARM and host computer using RS232 serial communication way to achieve, can guarantee the communication rate of $115 \sim 200$ bps to transmit the data accurately [11]. When the system receives the instruction PC data transmitted over the open system file system initialization, code analysis, 
interpolation and data processing system for reading instruction execute the corresponding operation system software, as shown in figure 3.

\section{Improved digital PID control algorithm and simulation}

Using the improved digital PID control, can achieve a certain range of no error control, improve the stability of the system PID, [12] algorithm for:

$\Delta u(k)=u(k)-u(k-1)=K_{p}[e(k)-e(k-1)]$
$K_{p}\left[\frac{T}{T_{t}} e(k)+\frac{T_{d}}{T} D e(k)-2 e(k-1)+e(k-2)\right]$

By the formula (11) can be obtained:

$$
\begin{aligned}
& \Delta u(k)=b_{0} e(k)+b_{1}^{e}(k-1)+b_{2} e(k-2) \\
& b_{1}=K_{p}\left(1+\frac{T}{T_{t}}+\frac{T_{d}}{T}\right)=K_{p}+T K_{I}+\frac{K_{D}}{T} \\
& b_{1}=-K_{p}-2 \frac{K_{D}}{T} \\
& b_{2}=K_{p} \frac{K_{D}}{T}
\end{aligned}
$$

The sampling period is the sampling period, and the sampling period is the output value, $k=0,1 \cdots, \boldsymbol{u}_{k}, K_{p}, K_{I}, K_{D}$ which is expressed as the proportional coefficient, integral coefficient and differential coefficient. For systems with large fluctuation range and rapid change, the general PID control can not achieve the desired results. Therefore, the text chooses the Ziegler-Nichols setting method to get the system parameters:

$K_{p}=7.728, K_{I}=0.031, K_{D}=0.00731$. The transfer function of the Ziegler-Nichols tuning method is assumed to be

$$
G_{0}(s)=\frac{4}{200 s+1} e^{-100 s}
$$

The simulation parameters of Ziegler-Nichols tuning method are shown in Table 1, and the simulation results are shown in Figure 4, Figure 5 and Figure 6.

\begin{tabular}{|c|c|c|c|}
\hline \multirow{2}{*}{ Simulation parameters } & \multicolumn{3}{|c|}{ Setting parameters } \\
\cline { 2 - 4 } & $K_{\mathrm{P}}$ & $K_{\mathrm{P}}$ & $K_{\mathrm{D}}$ \\
\hline $\mathrm{P}$ & $0.5 k_{\mathrm{P}}$ & & \\
\hline PI & $0.45 k_{\mathrm{P}}$ & $0.535 k_{\mathrm{P}} / T$ & $0.07 k_{\mathrm{P}} T$ \\
\hline PID & $0.6 k_{\mathrm{P}}$ & $1.2 k_{\mathrm{P}} / T$ & \\
\hline
\end{tabular}

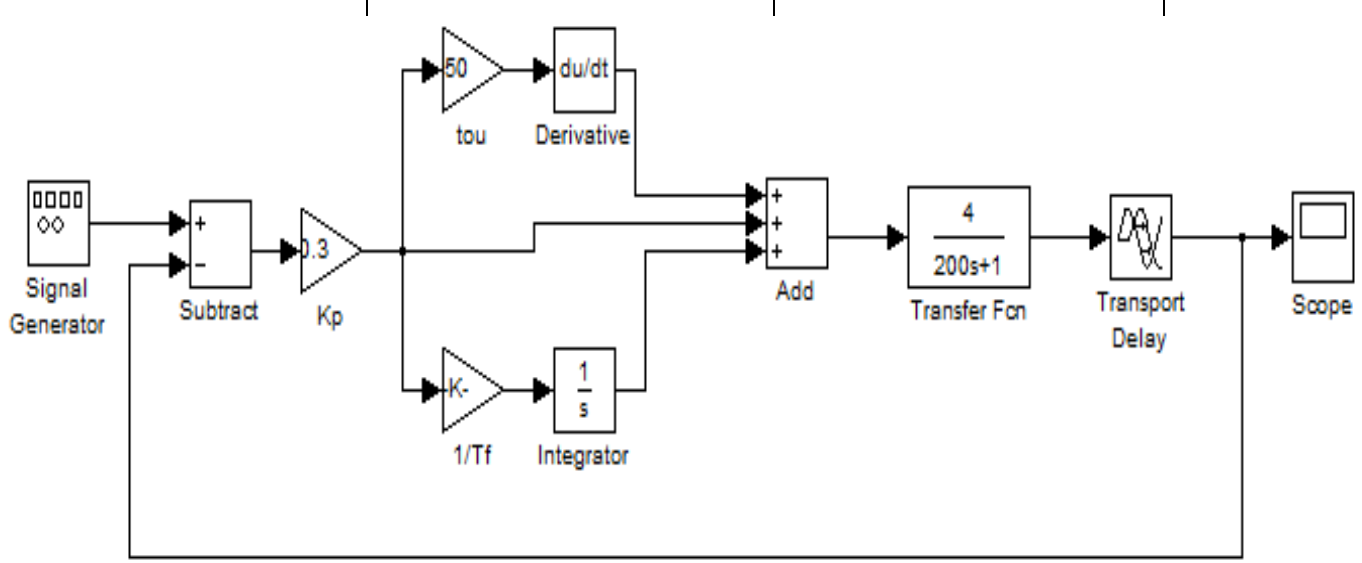

Fig. 4 Improved digital PID simulation diagram 
The improved digital PID, which is able to adapt to a wide range of fluctuations, rapid changes in the servo control system, and can maintain the stability of the general system, has a strong adaptability and versatility. By using MATLAB simulation, the results show that the interpolation algorithm can reduce the amount of interpolation computation and the debugging time, and reduce the overshoot, which provides a new method for the control of complex dynamic uncertain system.

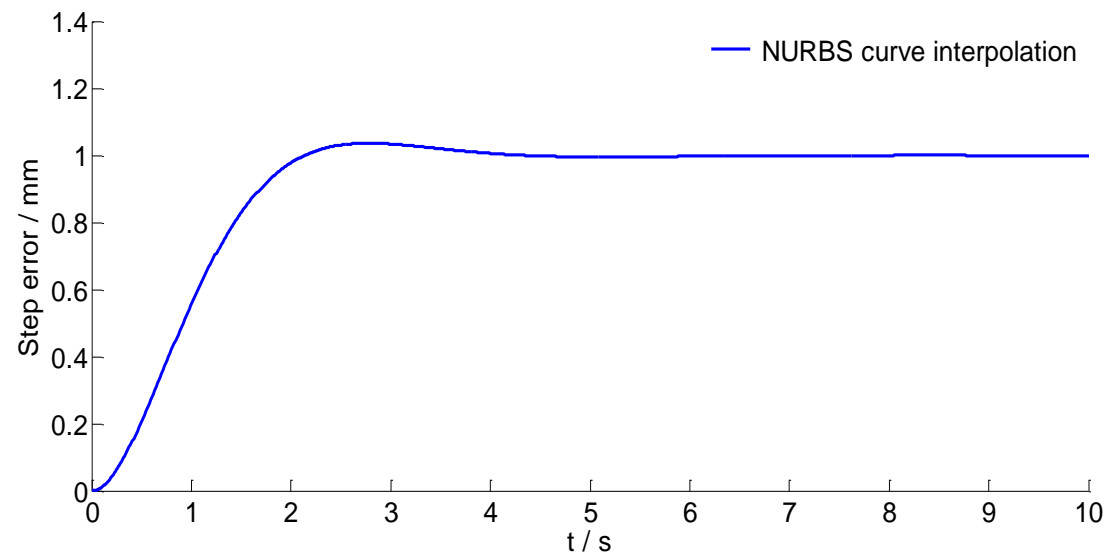

Fig. 5 NURBS curve interpolation

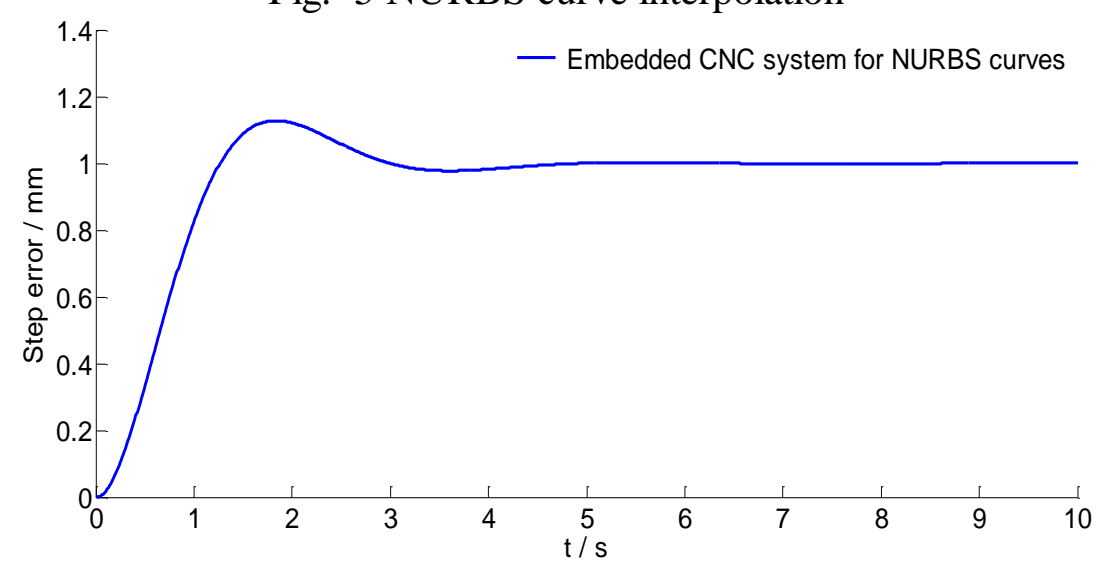

Fig. 6 Embedded CNC system for NURBS curve

\section{Summary}

(1) Based on NURBS curve interpolation algorithm, NURBS curve interpolation calculation and general computational complexity, proposed an improved interpolation algorithm, can reduce the time and improve the efficiency of interpolation interpolation.

(2) A new method of PID algorithm for servo system is presented, which is able to adapt to the control system with large fluctuation range and rapid change, and can keep the stability of the general system. Finally, using MATLAB simulation, the results show that the interpolation algorithm can reduce the amount of interpolation computation and the debugging time, and reduce the overshoot, which provides a new method for the control of complex dynamic uncertain system.

\section{Acknowledgements}

The authors thank the financial supports from National Natural Science Foundation of China(Grant no. 51165024) and Science and Technology Major Project of "High-grade NC Machine Tools and Basic Manufacturing Equipment" (2010ZX040001-181).

\section{References}

[1] Fang Yi-Xiang, Liu Wen-Xue. Based on the geometric properties of the cubic uniform B-Spline curve structure description[J]. Journal of engineering graphics, 2, pp.96-102, 2006. 
[2] Zhang Wan-Jun, Hu Chi-Bing, Zhang Feng, et al . Honing machine motion control card three B spline curve method of interpolation arithmetic for CNC system [J].Chinese Journal of Manufacturing Technology \& Machine Tool , 8(8), pp.40-43,August 2012.

[3] Zhang Wan-Jun, HU Chi-bing, WU Zai-xin, et,al. Research on modification algorithm of Three B Spline curve interpolation technology [J].Chinese Journal of Manufacturing Technology \& Machine Tool ,2 pp.141-143,Feburary 2013.

[4] Zhang Wan-Jun, Zhang Feng, Zhang Guo-hua. Research on a algorithm of adaptive interpolation for NURBS curve. [J].Applied Mechanics and Materials ,Vol. 687-691, pp.1600-1603, December 2014.

[5] Zhang Wan-Jun, Zhang Feng, Zhang Guo-hua. Research on modification algorithm of Cubic B-spline curve interpolation technology. [J].Applied Mechanics and Materials, Vol. 687-691, pp.1596-1599, December 2014.

[6] Zhang Wan-Jun, Zhang Feng, Zhang Wan-Liang. Research on a NURBS curve of timing / interrupt interpolation algorithm for CNC system [J].Chinese Journal of Manufacturing Technology \& Machine Tool , 4(4), pp.183-187,April 2015.

[7] Kong Fan-Guo, Hao Shang-Hua,ZhongYan-Zhi. NURBS curve interpolation algorithm to achieve the VC [J]. China new technology and new products, 17, pp.8-9, 2009.

[8] Ye Bo-Sheng, Yang Shu-Zi. CNC system in cubic B-Spline curve interpolation method [J]. China Mechanical Engineering, 9 (3) , pp. 42 - 43, 1998.

[9] Li He-Cheng, Wang Yu-Ping, An interpolation based genetic algorithm for sloving nonlinear bilevel programming problems.Chinese Journal of Computers, 31(6), pp.910-918, June 2008.

[10] Shpitalni M, Koren Y, Lo CC. Realtime curve interpolators. Computer- Aided Design, 26, pp.832-838, 1994.

[11] Zhang Wan-Jun, Zhang Feng, Zhang Wan-liang. Research on high-grade CNC machines tools CNC system for B-Spline curve method of High-speed real-time interpolation arithmetic [J]. Chinese Journal of Manufacturing Technology \& Machine Tool , 8(8), pp.172-176,August 2015.

[12] Huang JT, Yang DCH.A generalized interpolator for command generation of parametric curves in computer controlled machines. Japan/USA Symposium on Flexible Automation, 1(1),pp. 393-399, January1992.

[13] Lo CC,Chung CY.Curve generation and control for biaxial machine tools. J.CSME ,18, pp.175-182,1997.

[14] Zhang wanjun.A Newton iteration-Rapson of NC interpolation system [P]. China : 201520043 $082.8,2015$.

[15] Zhang wanjun. Based on b-spline curve real-time interpolation of CNC system at a high speed [P]. China : 201520069002.6,2015.

[16] Zhang wanjun. A kind of based on ARM + FPGA dual CPU of NC interpolation system [P]. China : 201520969163. 0,2016.

[17] Zhang wanjun. A control system of numerical control for interpolation PCI system [P]. China : 201620110057.1,2016.

[18] Sun, Shujie, Lin, Hu; Zheng, Liaomo. A real-time and look-ahead interpolation methodology with dynamic B-spline transition scheme for CNC machining of short line segments [J]. Internation journal of advanced manufacturing technology, 84 (3) , pp. 1359-1370, 2016.

[19] Duan, Molong, Okwudire, Chinedum. Minimum-time cornering for CNC machines using an optimal control method with NURBSparameterization

[J]. Internation journal of advanced manufacturing technology, 85 (3) , pp. 1405-1418, 2016.

[20]Liu Qiang,Liu Huan, Yuan Songmei.High Accurate Interpolation of NURBS Tool Path for CNC Machine Tools[J]. China journal of mechanical engineering, 29 (5) , pp. 911-920, 2016.

[21] Zhang Liqiang,Zhang, Kai Yan, Yecui.Local Corner Smoothing Transition Algorithm Based on Double Cubic NURBS for Five-axis Linear Tool Path[J].Strojniskivestnik-journal of mechanical engineering, 29 (5) , pp. 647-656, 2016(9). 